George Matalanis ORCID iD: 0000-0001-9235-1569

\title{
Trends \& Controversies in Type A Aortic surgery in the 21st Century - Branch first aortic arch replacement
}

Nisal Perera MBBS and George Matalanis FRACS

Department of Cardiac Surgery, Austin Hospital, Melbourne, AUSTRALIA

Corresponding Author:

A/Prof George Matalanis

Department of Cardiac Surgery

Austin Hospital

PO Box 5555,

Heidelberg, Victoria, 3084

Australia

Email: george.matalanis@austin.org.au

Conflict of Interest: None

Sources of Funding: None

Running Head: Branch First Technique

This is the author manuscript accepted for publication and undergone full peer review but has not been through the copyediting, typesetting, pagination and proofreading process, which may lead to differences between this version and the Version of Record. Please cite this article as doi: 10.1111/jocs.15037.

This article is protected by copyright. All rights reserved. 
Keywords: aorta and great vessels

Word Count: 2379

\begin{abstract}
Acute type A dissection (ATAAD) remains a morbid condition with reported surgical mortality as high as $26 \%$. We describe our surgical approach to ATAAD which avoids a traditional approach of ascending aorta and "hemi-arch" replacement utilising deep hypothermic circulatory arrest (DHCA) and an open distal anastomosis. We also discuss the indications for adjunct techniques such as the frozen elephant trunk or complete aortic repair with endovascular methods. Arch replacement using the "branch-first technique" allows for complete proximal aortic replacement and sets up for straightforward future distal aortic intervention.
\end{abstract}

\title{
Introduction
}

Acute Type A aortic dissection (ATAAD) is a condition associated with a mortality rate up to $26 \%$ (1) and significant morbidity. Despite advances in numerous aspects of cardiac surgery a traditional approach of ascending aorta and "hemi-arch" replacement utilising deep hypothermic circulatory arrest (DHCA) and an open distal anastomosis persists for the management of this condition in most centres. We hereby describe the rationale behind our surgical approach of the branch-first total aortic arch replacement technique for the management of ATAAD.

\section{Operative Technique}

We have described our technique for branch first arch replacement in ATAAD previously (2). The essential aspects of our technique are listed below.

This article is protected by copyright. All rights reserved. 
Interrogation of pre-operative imaging

A computed tomography (CT) aortogram is nearly always available. Note is made of the position, size and the relationships of the true lumen (TL) and false lumen (FL) along the entire aorta. Each branch vessel is inspected for dissection and obstruction. Entry and re-entry tears are noted.

\section{Monitoring and positioning}

Bilateral radial or brachial arterial lines are inserted. A femoral arterial line is placed prior to draping, or it can be placed by the surgeon once the operative field is prepared. Cerebral monitoring is carried out by a combination of electroencephalogram bispectral index (BIS), near-infrared spectroscopy (NIRS) and transcranial doppler ultrasonography (TCD). The patient is draped widely to allow access to both groins, the neck, and the anterior chest.

\section{Cannulation and establishment of cardiopulmonary}

In the majority of cases, cardiopulmonary bypass (CPB) is instituted by femoral arterial inflow and central venous drainage. When the femoral arteries are dissected on the preoperative imaging or have a very weak pulse an alternative arterial cannulation site is used. Options include direct aortic cannulation using a seldinger technique under trans oesophageal echocardiography (TOE) guidance or, right or left axillary cannulation using an $8 \mathrm{~mm}$ side graft. It is vital to be alert to the risk of malperfusion during establishment of CPB, which may occur not only with femoral but with any source of arterial inflow. Initially partial bypass is established to keep the heart ejecting to maintain TL flow. Multiple pressures are monitored for large differentials, which then mandate the need for another inflow source. Aortic 
insufficiency is managed using a left ventricular vent through the right superior pulmonary vein. Core cooling is commenced to $25^{\circ} \mathrm{C}$, but this is performed slowly initially until the debranching process is complete. This reduces the risk of ventricular fibrillation and distension which would require earlier than planned aortic cross clamping and cardioplegic arrest.

\section{Branch first arch replacement}

The innominate artery (IA), Left Carotid artery (LCCA) and occasionally the Left Subclavian artery (LSA) are exposed for a length of $3 \mathrm{~cm}$. In the absence of cardiogenic shock, a significant component of the mobilisation can be done before the onset of CPB. The innominate artery is clamped just proximal to its bifurcation and about $1 \mathrm{~cm}$ distal to its origin from the arch and transected between the clamps. The distal stump is then anastomosed to the first limb of the trifurcation arch graft with a side-arm perfusion port (TAPP graft, Vascutek Ltd., Renfrewshire, Scotland, UK) in an end-to-end fashion. After thorough de-airing, clamps are rearranged to allow antegrade flow to resume via the side-arm of the TAPP graft which is connected to a separate "cerebral circuit", powered by its own pump. It is important to note that this manoeuvre secures flow to the true lumen of this vessel. Hence the risk of cerebral malperfusion is obviated very early in the course of the operation. This situation is quite different from that of carotid endarterectomy, where the distal common and external carotid arteries are clamped, and there is complete reliance on an intact circle of Willis for the sole collateral supply to the ipsilateral hemisphere. The very proximal position of the IA clamp allows the rich collateral networks around the head and neck, fed from ongoing perfusion of the other two 
arch branches and returning to the right carotid via its external carotid branch, to augment right hemispheric perfusion over and beyond that provided by the circle of Willis. Furthermore, typically only short clamp times (around 10 minutes) are required, in contrast with unilateral antegrade perfusion where the same collateral network is utilized but the exclusion time is for the whole duration of arch reconstruction.

With the debranched IA out of the field, there is further improvement in exposure to the LCCA. The LCCA can be mobilized, reconstructed and perfused in the same sequence to the second limb of the TAPP graft. Again, the same rich network is utilized to maintain left hemispheric perfusion, for the even shorter clamp time for this smaller anastomosis.

If there is adequate exposure to the left subclavian artery (LSCA) it is mobilized and clamp isolated, with the assistant helping to ease it out of the depth of the surgical field by gentle upward traction on the clamp. The LSCA is reconstructed and perfused to the third limb of the TAPP graft. Otherwise, we delay reconstruction of the LSCA until after institution of distal circulatory arrest, when significant space is created by the total decompression of the distal arch.

Importantly, throughout the debranching process, perfusion is not interrupted to the heart or distal organs. The branched trifurcation graft maintains continuous antegrade cerebral perfusion throughout the whole of the rest of the procedure, until completion of its anastomosis to the ascending aortic graft, when regular inflow can take over.

At this stage, the perfused TAPP graft can easily be laid out of the field, over the patient's neck. Distal circulatory arrest is commenced, and cardioplegia instituted. An 
open distal anastomosis can then be constructed. The use of an Anteflow graft (Vascutek Ltd., Renfrewshire, Scotland, UK) allows transfer of femoral inflow to the central aorta, and re-establishment of antegrade body flow.

Where possible, we resuspend the aortic valve and perform a simple proximal anastomosis. If the root is extensively dissected or aneurysmal then a formal root reconstruction is performed either as a valve sparing or Bentall reconstruction as appropriate. The common stem of the TAPP graft is then anastomosed to the proximal ascending graft. Positioning of the common stem of the TAPP graft anastomosis proximally allows for a long landing zone, should a second stage endovascular reconstruction be required.

In a third of cases we use a frozen elephant trunk (FET) and/or completion stent grafting. Completion stent grafting involves proximal descending aortic endografting and distal aortic relamination with balloon assisted intimal disruption and bare metal stenting (STABILISE), as described elsewhere for Total Aortic Repair(3).

We do not routinely use the FET technique as it introduces the potential increased risk of paraplegia. However, we consider FET use in ATAAD with a large proximal descending entry beyond reach of arch resection, often associated with distal TL collapse, distal malperfusion and retrograde Type A dissection. We also use a FET when the distal aorta is particularly fragile, to spread the load of distal anastomotic purchase. Finally, we also consider it if planning for an open rather than endovascular second stage operation on the descending aorta, to avoid difficult cross clamping in the region of adhered recurrent laryngeal nerve or pulmonary artery 
We consider early or late completion thoracoabdominal stenting in the setting of ongoing or recurrent distal malperfusion, radiological TL collapse, rapid dilatation of the FL and concave TL which is thought to be a marker of compromised TL perfusion with the potential for future organ ischemia.

\section{Discussion}

There are numerous, seemingly sound arguments against performing a total arch replacement in ATAAD. An ascending or hemi arch replacement is thought by many to be the safest and most expeditious procedure to perform in the acute situation. This is because the arch is rarely thought to be the site of the primary tear. It avoids doing a difficult zone 2 or 3 anastomosis deep in the chest which can result in difficult to reach bleeding anastomotic sites, and left recurrent laryngeal nerve injury. Circulatory arrest time is reduced by doing a limited operation. Late distal aortic problems are believed to be uncommon and if they do arise they can potentially be treated using an endovascular technique. Finally performing an aortic arch replacement is not in the armamentarium of junior or non-aortic surgeons. However, in our experience, all of these assertions are not completely accurate.

The mortality rate for treated ATAAD remains up to $26 \%$ in the IRAD database(1). It may be legitimately argued that mortality will necessarily be higher in acute dissection due to the not infrequent occurrences of organ malperfusion or shock. Nevertheless, in a recent study where subgroup analysis was performed, overall mortality was $19 \%$, but still remained at $14 \%$ in the lowest risk group (4). Of note, only $5 \%$ of cases in that series received total arch replacements. In our experience with total arch replacement in 39 cases, we had an overall 5(12.8\%) hospital 
mortalities and $2(5.1 \%)$ strokes. However, there were no deaths or strokes in patients without pre-operative organ malperfusion or shock. Of the 5 mortalities, 2 presented with frank rupture, 2 with gut ischaemia and 1 with myocardial infarction. We believe that our technique results in better early outcomes for a number of reasons. These include avoidance of cerebral circulatory arrest, and reduced cardiac and distal organ ischaemic time. More haemostatic anastomoses performed in unhurried fashion and in good quality tissue. Removal of all large proximal tears not only in the ascending, but also in the arch and proximal descending aorta, reduces persistent/recurrent false lumen pressurisation. This not only supports secure suture line haemostasis, but also promotes distal false lumen thrombosis. The advantages of moving the distal anastomosis forwards to Zone 1 or 2, such as preservation of the recurrent nerve and easier access, are equally applicable to our technique.

In cases, who have needed further downstream aortic reconstruction, the procedure was greatly facilitated by 2 factors inherent to our technique compared to that of the hemiarch. Firstly, location of the origin of the trifurcation graft from the proximal neo-ascending aorta, leaves considerable "landing zone" for endograft sealing or aortic clamping in open cases. Secondly, as all the arch branches are securely attached by Dacron grafts to the neo-ascending aorta, there is no need to be concerned about complex branched arch endografts to avoid endoleaks related to proximity to branches.

By contrast, in the situation of a previous hemi arch replacement, simple endovascular stenting cannot cover tears in the native arch for fear of concurrent coverage of the innominate or left common carotid arteries. It can also create further intimal tears, which did not exist before, at the proximal end of the stent. This off course is obviated 
when stenting into the long dacron landing zone created by a branch first arch replacement.

As ATAAD is a dynamic process, pre-operative CT aortography cannot definitively localise all entry and re-entry tears that will be discovered at the time of surgery. There is a $23 \%$ risk of finding the primary tear in the arch when opening and excising the ascending aorta under DHCA (6). A surgeon not familiar with arch surgery will either choose to ignore this finding or perform a hurried arch replacement in an environment of an inexperienced surgeon and more importantly support from unprepared anaesthesia, perfusion and nursing team.

Leaving a large tear in the arch predisposes to early aneurysmal dilation and/or rupture due to persistent pressurization of the false lumen as discussed above. On the other hand, unexpected hemi-arch or total arch replacement can increases the complexity, time, haste and anxiety in the hands of the junior or non-aortic surgeon leading to suboptimal results. At our institution, and now many other in Australia and New Zealand, routine use and teaching of trainees of the branch first technique, in both elective and urgent cases, has increased confidence and reduced anxiety in treating ATAAD. We hope that widespread adoption of our approach will lead to improved early and late outcomes of this deadly condition.

\section{Conclusion}

The use of a branch first aortic arch replacement technique in ATAAD results in improved acute outcomes such as bleeding, malperfusion and death. It secures true lumen flow to cerebral vessels, increases the chance of false lumen thrombosis and it 
simplifies future intervention. This approach is applicable to all cardiac surgeons not only the aortic sub specialist.

\section{References}

1. Berretta P, Patel HJ, Gleason TG, Sundt TM, Myrmel T, Desai N, et al. IRAD experience on surgical type $A$ acute dissection patients: results and predictors of mortality. Ann Cardiothorac Surg. 2016;5(4):346-51.

2. Galvin SD, Perera NK, Matalanis G. Surgical management of acute type A aortic dissection: branch-first arch replacement with total aortic repair. Ann Cardiothorac Surg. 2016;5(3):236-44.

3. Hofferberth SC, Nixon IK, Boston RC, McLachlan CS, Mossop PJ. Stent-assisted balloon-induced intimal disruption and relamination in aortic dissection repair: the STABILISE concept. J Thorac Cardiovasc Surg. 2014;147(4):1240-5.

4. Olsson C, Hillebrant CG, Liska J, Lockowandt U, Eriksson P, Franco-Cereceda A. Mortality in acute type A aortic dissection: validation of the Penn classification. Ann Thorac Surg. 2011;92(4):1376-82.

5. Sobocinski J, Patterson BO, Karthikesalingam A, Thompson MM. The Effect of Left Subclavian Artery Coverage in Thoracic Endovascular Aortic Repair. Ann Thorac Surg. 2016;101(2):810-7.

6. Lansman SL, McCullough JN, Nguyen KH, Spielvogel D, Klein JJ, Galla JD, et al. Subtypes of acute aortic dissection. Ann Thorac Surg. 1999;67(6):1975-8; discussion 9-80.

This article is protected by copyright. All rights reserved. 


\section{University Library}

\section{- M M N E R VA A gateway to Melbourne's research publications}

Minerva Access is the Institutional Repository of The University of Melbourne

Author/s:

Perera, N;Matalanis, G

Title:

Trends and controversies in type A aortic surgery in the 21st century: Branch first aortic arch replacement

Date:

2020-09-29

\section{Citation:}

Perera, N. \& Matalanis, G. (2020). Trends and controversies in type A aortic surgery in the 21st century: Branch first aortic arch replacement. JOURNAL OF CARDIAC SURGERY, 36 (5), pp.1766-1769. https://doi.org/10.1111/jocs.15037.

Persistent Link:

http://hdl.handle.net/11343/276370 